\title{
Plateletpheresis Donation Trend and a Ban on Family/Replacement Donation in China: A Pseudo-Panel Data Approach Analysis
}

\author{
Jinyan Chen \\ Guangzhou Blood Center \\ Xuemei Fu \\ Chengdu Blood Center \\ Shijie Li \\ Guangzhou Blood Center \\ Ying Li \\ Chengdu Blood Center \\ Jiankun Kang \\ Chengdu Blood Center \\ Huiyou Chen \\ Guangzhou Blood Center \\ Liqiao Zhou \\ Guangzhou Blood Center \\ Yongshui Fu \\ Guangzhou Blood Center
}

Guoli Zhou ( $\square$ zhoug@msu.edu)

Michigan State University https://orcid.org/0000-0002-4497-5366

\section{Research}

Keywords: plateletpheresis donation, ban on family/replacement donation, pseudo-panel data approach, piecewise linear mixed model

Posted Date: September 2nd, 2020

DOI: https://doi.org/10.21203/rs.3.rs-67553/v1

License: (c) (1) This work is licensed under a Creative Commons Attribution 4.0 International License. Read Full License 


\section{Abstract}

Background A nationwide ban on family/replacement donation (FRD) went into effect on April 1, 2018 in China. To date, no reports relevant to the trend of plateletpheresis donations before and after a nationwide ban on FRD were found.

Methods We used two independent full samples, consisting of 135851 and 82129 plateletpheresis donors from Guangzhou and Chengdu between October 2012 and September 2019, respectively. A pseudo-panel dataset approach was applied by grouping three time-invariant covariates - gender, blood donation history, and birth year across 14 cross-sections (a 6-month interval each)to form a total of 24 cohort groups $(14 \times 24=336$ cohorts, i.e., cells) with each having common covariates. The outcome was average apheresis platelet units per donor in each cell. We performed a two-piecewise linear mixed model with the cross-section (i.e., time) just right before the ban as a time breakpoint(i.e., 11th cross-section) to examine the trend of outcome with the adjustment of three time-invariant covariates. We removed the family/replacement donations in each of the first 11 cross-sections to detect its possible influence on the trend.

Results The final model for the samples from Guangzhou presented a two-piecewise linear trend of the outcome over time with a horizontal line to the left of the breakpoint ( $\beta$ timeBefore $11=0.0111, p=0.0976$ ) and a significantly positive linear trend to the right ( $\beta$ timeAfter $11=0.0404, p<0.0001)$. The male donors and the donors with plateletpheresis donation history had an increased baseline outcome and a significant outcome change over time after the ban. Such a twopiecewise linear trend pattern can be replicated using the samples from Chengdu with some minor variations. Removing the FRD before the ban can change the pattern.

Conclusion The significant increase of the average apheresis platelet units per donor over time after the FRD ban may be related to the implement of the FRD ban and the improved donation behavior of male donors and/or donors with platelet donation history after the ban. Our findings may potentially motivate the policymakers in other countries where the FRD for plateletpheresis donation is still legitimate to phase out their FRD strategy and ultimately achieve $100 \%$ voluntary plateletpheresis donation.

\section{Contributions To The Literature}

It was thought that banning the family/replacement donation (FRD) could cause severe blood shortage in countries with limited resources. However, a phenomenon of "fewer donors, more blood" in plateletpheresis donation practice since the implementation of the FRD ban in China has broken this belief.

To date, no evidence-based research relevant to the FRD ban has been found. It is necessary to evaluate the influence of the FRD ban on plateletpheresis donation in China.

Ours findings filled the literature gap and motivated the policymakers to change the FRD policy for plateletpheresis donation in those countries where the FRD is still legitimate.

\section{Background}

The adequacy and safety of blood supply is a major public health challenge in the world[1,2]. There is an ongoing debate over the family/replacement donation (FRD) policy influencing both shortage and safety of blood supply [3-5]. FRD, also called mutual donation, occurs when family members are required to donate blood to replace each unit used by their friend or relative[6] Currently, the FRD is legitimate and considered to be indispensable to the transfusion services in many countries with the limited resources[7-9]. Although this type of blood donation may provide short-term solutions for dealing with the shortage of blood supply[10], it increases public distrust in voluntary blood donation and affects the quality and safety of donated blood[11]. Phasing out the FRD is one of the targets in a global framework for action to achieve $100 \%$ voluntary blood donation developed by the World Health Organization and the International Federation of Red Cross and Red Crescent Societies[9]. A nationwide ban on the FRD went into effect on April 1, 2018 in China. With the "more donors, more blood " belief, it was believed that banning the FRD would cause serious consequences related to the shortage of blood supply[8, 12]. However, a phenomenon of "fewer donors, more blood" for the plateletpheresis donation has been emerging in the field since the implement of the FRD ban in China. To date, no reports relevant to the trend of plateletpheresis donations before and after a nationwide ban on family/replacement donation were found. Therefore, it is necessary to quantify the evidence-based trend of the plateletpheresis donation and the potential contributing factors to this trend before and after the FRD ban in China.

In present study, we examined a model-based trend of the apheresis platelet units donated before and after the FRD ban using two independent full samples in China with a pseudo-panel data approach followed by a piecewise linear mixed model.

\section{Methods}

\section{Study population and study design}

Based on the availability of the data, we used two independent full samples between October 1, 2012 - September 30, 2019 from Guangzhou Blood Center, the second largest blood center in China and Chengdu Blood Center, the largest blood centers in Western China, which were named as "discovery GZ set" and "replicate CD set" at the individual level, respectively. The discovery and replicate sets consisted of 135851 and 82129 plateletpheresis donors, respectively. We enrolled the donors with an age of $18 \sim 60$ years old, weight $\geq 50 \mathrm{~kg}$ for male and $\geq 45 \mathrm{~kg}$ for female, systolic blood pressure $90 \sim 140 \mathrm{mmHg}$ and diastolic blood pressure $60 \sim 90 \mathrm{mmHg}$, pulse $60 \sim 100$ beats/min, normal body temperature, platelet counts before donation $150 \sim 450 \times 109 / \mathrm{L}$, and no any other health conditions according to the China national standard for the eligible donor selection criteria (GB 18467 - 2011). All data used in the present study were de-identified. 
We chose 5.5 years and 1.5 years before and after April 1, 2018, respectively, to set our investigation time window with a six-month interval as a cross-section, generating a total of 14 repeated cross-sections with 11 and 3 cross-sections before and after the ban, respectively. Based on the GB $18467-2011$, a donor may donate up to a total of 24 plateletpheresis collections during a 12-month rolling period. Thus, some plateletpheresis donors may appear more than once across the 14 cross-sections, but not all donors appear in every cross-section. Therefore, there were some different degrees of the correlations of the data across 14 cross-sections, and our datasets presented a pseudo-panel structure[13] .

\section{Measurements of outcome variable and covariates}

The records for the total amount of the platelets donated by each donor within each cross-section, grouping covariates - gender, birth year, and blood donation history (see detailed information below), as well as the variable -family/replacement platelet donation (yes vs. no) were extracted from the archived blood donation documents in both blood centers. The outcome variable at the cohort (i.e., cell) level within the pseudo-panel datasets (see detailed information below) was defined as average platelet units per donor in each cell.

\section{Construction of pseudo-panel datasets}

The pseudo-panel data approach is actually a solution to transform the individual-level cross-sectional data into the group-level data (i.e., pseudo-panel data)such that the typical longitudinal models can be applied to efficiently and consistently estimate the change of the interested outcome variable over time. [13] This approach has been increasingly applied to public health[14, 15] .

According to the methods described in the literature[16, 17], we constructed two pseudo-panel datasets from our discovery and replicate data by grouping three time-invariant variables -gender, birth year, and blood donation history. Other potential covariates such as ethnicity, occupation, and education with large proportions of missing data were excluded from the analyses. Briefly, individual platelet donors were first classified based on gender that had two categories male and female. To balance the size of each cohort ( $\geq 100$, named as large cohort) and the number of large cohorts[16] within our pseudo-panel datasets, we defined birth year as three categories - "1952-1974", "1975-1984", and "1985-2001". The variable blood donation history was coded as 4 levels - "None", "Whole Blood Donation Only" (abbreviated to "WB"), "Apheresis Platelet Donation Only" (abbreviated to "PLT"), and "Both WB and PLT Donations" (abbreviated to "Both"). The individuals were then further divided by these two variables, generating $2 \times 3 \times 4=24$ cohort groups across the 14 cross-sections, i.e., $24 \times 14=$ 336 cells for each of two pseudo-panel datasets. The generated each cohort group had common gender, birth year, and blood donation history. To reduce the measurement error[17], we removed the cells with less than 30 individual donors in each dataset to generate two final pseudo-panel datasets, named as "overall Guangzhou pseudo-panel set" (abbreviated to "overall GZ set") ( $n=330$ cells) and "overall Chengdu pseudo-panel set" (abbreviated to "overall CD set") $(n=316$ cells), respectively, for further analyses. More detailed information about the summaries of the constructed pseudo-panel datasets is presented in Table 4.

Our study was actually an observational study using pseudo-panel approach to analysis the data[13-15]. Thus, we used the Strengthening the Reporting of Observational Studies in Epidemiology (STROBE) statement to ensure standardisation and enhance the quality of the reporting[18]. See Additional file 1 for the STROBE statement.

\section{Statistics}

For the individual-level data, we used two-tailed independent t-tests $(\alpha=0.05)$ to compare total number of donors, total number of donations, and total apheresis platelet units $(U)$, average plateletpheresis donations per donor, and average apheresis platelet units (U) per donor between before and after the ban (also called "after-before mean difference"). To compare two after-before mean differences between groups within each covariate, we applied two-tailed Z-test ( $Z=($ mean difference1-mean difference 2$) /$ sqrt(se12 + se22), $a=0.05)$.

For overall GZ pseudo-panel set, we plotted outcome values (i.e., average apheresis platelet units per donor per cell) from each cohort group that had common gender, birth year, and blood donation history versus time (i.e., 14 cross-sections) as well as overall average outcome values from all 24 cohort groups versus time to visualize whether the trend of the outcome over time is linear or non-linear. Given that generalized linear mixed model and mixed-generalized ordered logit model have been successfully applied to analyze the trend of the outcome variable (proportion or probability) over time within a pseudo-panel dataset[14, 19], for our pseudo-panel dataset with a continuous outcome variable, we applied a linear mixed model for modelling the linear trend, or modelling the nonlinear trend using a piecewise linear mixed model with the defined time (i.e., cross-section) breakpoint(s) based on the above-mentioned visualization[20]. We conducted a model selection starting from an unadjusted model (i.e., model 1 - pure time trend model without any covariates) to an adjusted model (i.e., model 2 = model $1+$ significant covariates), and then to a final model (i.e., model $3=$ model $2+$ significant interaction terms). To compare two raw regression coefficients within the same final model, we used one-tailed Z-tests $[Z=a b s(\beta 2-\beta 1) /$ sqrt(SE12 + SE22), $\alpha=0.05]$.

The assumptions of normality and homoscedasticity for piecewise linear mixed effects models were examined by visualizing marginal (for fixed effects only) and conditional (for both fixed and random effects) Pearson residual plots.

For five-fold cross-validation of the final model, the data were randomly split into 5 roughly equal-sized subsets using SAS PROC SURVEYSELECT procedure, the final model was fitted to the 4 subsets of the data using SAS PROC MIXED procedure with the STORE statement. The prediction error - Root Mean Square Error (RMSE) of the fitted model to predict the fifth subset using SAS PROC PLM procedure was calculated. This procedure was repeated 5 times such that each subset was used for testing exactly once and an average RMSE of 5-fold cross-validation was calculated by using the formula: SQRT((RMSE12 + RMSE22 + ... RMSE52)/5) and further compared with that from the model-fitting using the full sample to determine if there was an over-fitting issue. 
To test for replication of the trend of the overall outcome mean over time obtained from the overall GZ set, we applied the same methods as described above to the independent pseudo-panel dataset from Chengdu Blood Center, i.e., overall CD set.

To detect the potential effect of family/replacement plateletpheresis donations on the model-based trend of the average apheresis platelet units per donor over time identified from the overall GZ and CD sets, we removed the family/replacement plateletpheresis donations in each of the first 11 cross-sections (all donations after the ban were voluntary) from the overall individual-level data. Then we re-grouped the remaining individuals (81801 and 48768 voluntary plateletpheresis donors remaining from the discovery and replicate sets, respectively)to generate two nested pseudo-panel subsets, named as "voluntary GZ subset" $(n=300$ cells) and "voluntary CD subset" ( $n=284$ cells), respectively. Finally, we used the same methods as described above to fit the piecewise linear mixed models for both subsets.

All data management and statistical analyses described above were conducted with R (R Development Core Team) and SAS v9.4 (SAS Institute, Cary, North Carolina).

\section{Results}

Demographics of plateletpheresis donors across 14 successive cross-sections with a 6-month interval each in both overall discovery and replicate sets

Table 1 and Additional file 2 showed a similar demographics of plateletpheresis donors across 14 successive cross-sections between overall discovery and replicate sets. Our overall discovery set consisted of $69.3-80.3 \%$ of plateletpheresis donors who were male across all 14 cross-sections, $5.0-6.5 \%$ and $8.8-$ $9.7 \% \geq 46$ years old across the first 11 cross-sections (i.e., before the ban) and the 12 th -14 th cross-sections (i.e., after the ban), respectively, as well as $45.1-$ $51.8 \%$ of donors who had a blood donation history (14.4-14.5\%: WB only, 18.6-21.9\%: PLT only, and 12.0-15.5\%: Both) across the cross-sections before the ban and 74.8-76.8\% with a blood donation history (17.5-20.9\%: WB only, 27.9-28.5\%: PLT only, and 25.4-31.5\%: Both) across the cross-sections after the ban (Table 1). The overall GZ set also contained about 15.4-41.9\% of FRDs across the first 11 cross-sections (for the 12th - 14th cross-sections, no donations were FRD) (Table 1). In the overall replicate set, about $63.3-75.5 \%$ were male across all 14 cross-sections; $10.0-13.9 \%$ and $16.3-17.9 \%$ were $\geq$ 46 years old across the first 11 cross-sections and the 12 th -14 thcross-sections, respectively; $30.1-40.6 \%$ and $63.9-72.4 \%$ had a blood donation history across the cross-sections before and after the ban, respectively; and 3.9-52.6\% were family/replacement donors across the first 11 cross-sections (Additional file 2). 
Table 1

Demographics of individual plateletpheresis donors across 14 successive cross-sections in Guangzhou Blood Center $(\mathrm{N}=135851)$

\begin{tabular}{|c|c|c|c|c|c|c|c|c|c|c|c|c|c|}
\hline & $\begin{array}{l}2012 / 10 \\
-2013 / 3\end{array}$ & $\begin{array}{l}2013 / 4 \\
-2013 / 9\end{array}$ & $\begin{array}{l}2013 / 10 \\
-2014 / 3\end{array}$ & $\begin{array}{l}2014 / 4 \\
-2014 / 9\end{array}$ & $\begin{array}{l}2014 / 10 \\
-2015 / 3\end{array}$ & $\begin{array}{l}2015 / 4 \\
-2015 / 9\end{array}$ & $\begin{array}{l}2015 / 10 \\
-2016 / 3\end{array}$ & $\begin{array}{l}2016 / 4 \\
-2016 / 9\end{array}$ & $\begin{array}{l}2016 / 10 \\
-2017 / 3\end{array}$ & $\begin{array}{l}2017 / 4 \\
-2017 / 9\end{array}$ & $\begin{array}{l}2017 / 10 \\
-2018 / 3\end{array}$ & $\begin{array}{l}2018 / 4 \\
-2018 / 9\end{array}$ & $\begin{array}{l}2018 \\
-201\end{array}$ \\
\hline \multicolumn{14}{|l|}{ Gender } \\
\hline \multirow{2}{*}{$\begin{array}{l}\text { Male, } \mathrm{n} \\
(\%)\end{array}$} & 5409 & 6176 & 5944 & 6938 & 7371 & 8117 & 8395 & 8968 & 8742 & 9274 & 9042 & 4857 & 590 \\
\hline & $(71.0)$ & (71.5) & $(69.3)$ & (71.3) & $(72.1)$ & $(73.0)$ & (73.3) & (77.9) & (77.0) & (80.3) & (78.7) & (74.9) & (75.2 \\
\hline \multirow{2}{*}{$\begin{array}{l}\text { Female, } \\
\mathrm{n}(\%)\end{array}$} & 2210 & 2458 & 2638 & 2790 & 2858 & 2995 & 3062 & 2542 & 2604 & 2282 & 2446 & 1624 & 1944 \\
\hline & $(29.0)$ & $(28.5)$ & (30.7) & $(28.7)$ & $(27.9)$ & $(27.0)$ & $(26.7)$ & (22.1) & $(23.0)$ & (19.7) & (21.3) & $(25.1)$ & $(24 . \varepsilon$ \\
\hline \multicolumn{14}{|l|}{ Age } \\
\hline \multirow{2}{*}{$\begin{array}{l}\leq 35 y, n \\
(\%)\end{array}$} & 5993 & 6702 & 6698 & 7414 & 7812 & 8523 & 9237 & 9110 & 9055 & 9136 & 9071 & 4722 & 5966 \\
\hline & (78.7) & (77.6) & $(78.0)$ & (76.2) & (76.4) & (76.7) & (80.6) & (79.1) & (79.8) & $(79.1)$ & (79.0) & (72.9) & (76.) \\
\hline \multirow{2}{*}{$\begin{array}{l}>35 y, n \\
(\%)\end{array}$} & 1626 & 1932 & 1884 & 2314 & 2417 & 2589 & 2220 & 2400 & 2291 & 2420 & 2417 & 1759 & 188 \\
\hline & (21.3) & $(22.4)$ & $(22.0)$ & $(23.8)$ & $(23.6)$ & (23.3) & (19.4) & (20.9) & (20.2) & (20.9) & $(21.0)$ & $(27.1)$ & (24.) \\
\hline \multicolumn{14}{|c|}{$\begin{array}{l}\text { Blood donation } \\
\text { historya }\end{array}$} \\
\hline \multirow{2}{*}{$\begin{array}{l}\text { None, } \mathrm{n} \\
(\%)\end{array}$} & 3791 & 4166 & 4581 & 5134 & 5615 & 5985 & 6308 & 6129 & 5945 & 5575 & 5718 & 1631 & 2465 \\
\hline & $(49.8)$ & (48.3) & $(53.4)$ & $(52.8)$ & (54.9) & (53.9) & (55.1) & (53.2) & (52.4) & $(48.2)$ & $(49.8)$ & $(25.2)$ & (31.5 \\
\hline \multirow{2}{*}{$\begin{array}{l}\text { WB, n } \\
(\%)\end{array}$} & 906 & 1331 & 1132 & 1519 & 1484 & 1875 & 1810 & 1831 & 1660 & 1665 & 1560 & 1352 & 1425 \\
\hline & (11.9) & (15.4) & (13.2) & (15.6) & (14.5) & (16.9) & (15.8) & (15.9) & (14.6) & $(14.4)$ & (13.6) & (20.9) & (18.2 \\
\hline \multirow{2}{*}{$\begin{array}{l}\text { PLT, n } \\
(\%)\end{array}$} & 1793 & 1925 & 1755 & 1880 & 1899 & 1941 & 1971 & 2098 & 2153 & 2526 & 2373 & 1849 & 187 \\
\hline & $(23.5)$ & $(22.3)$ & $(20.5)$ & (19.3) & (18.6) & (17.5) & $(17.2)$ & (18.2) & (19.0) & (21.9) & (20.7) & $(28.5)$ & $(23 . \varsigma$ \\
\hline \multirow{2}{*}{$\begin{array}{l}\text { Both, } n \\
(\%)\end{array}$} & 1129 & 1212 & 1114 & 1195 & 1231 & 1311 & 1368 & 1452 & 1588 & 1790 & 1837 & 1649 & $207 !$ \\
\hline & $(14.8)$ & $(14.0)$ & $(13.0)$ & (12.3) & $(12.0)$ & (11.8) & (11.9) & (12.6) & $(14.0)$ & (15.5) & (16.0) & $(25.4)$ & $(26.4$ \\
\hline \multicolumn{14}{|c|}{ FRD in current interval } \\
\hline \multirow{2}{*}{$\begin{array}{l}\text { Yes, n } \\
(\%)\end{array}$} & 2194 & 3690 & 3654 & 4898 & 5865 & 6720 & 6633 & 7840 & 7435 & 8485 & 3677 & 0 & 0 \\
\hline & $(15.4)$ & $(24.1)$ & $(24.2)$ & $(28.9)$ & $(34.2)$ & $(36.1)$ & $(34.7)$ & $(40)$ & $(39.2)$ & $(41.9)$ & $(18.5)$ & $(0.0)$ & $(0.0)$ \\
\hline \multirow{2}{*}{$\begin{array}{l}\text { No, } n \\
(\%)\end{array}$} & 12031 & 11634 & 11446 & 12062 & 11303 & 11910 & 12498 & 11765 & 11513 & 11750 & 16176 & 17742 & 220 \\
\hline & $(84.6)$ & $(75.9)$ & $(75.8)$ & (71.1) & $(65.8)$ & $(63.9)$ & $(65.3)$ & $(60)$ & $(60.8)$ & $(58.1)$ & $(81.5)$ & $(100.0)$ & $(100$ \\
\hline
\end{tabular}

Bold cross-sections denote the ones after the ban on FRD.

a"None"=no blood donation history; "WB"=whole blood donation history only; "PLT"=plateletpheresis donation history only; "Both"=both whole blood and plate donations history.

In either discovery or replicate dataset, both average total number of donations and average total apheresis platelet units were significantly increase dafter the ban compared to those before the ban; in contrast, the change of average total number of donors from before to after the ban was the opposite $(t=2.42-5.56$, $p=0.0325-0.0001$ ) (Table 2, Additional file 3 and Additional file 4). These results indicated that there is a phenomenon of" fewer donors, more blood" after the FRD ban. 
Table 2

Comparisons of total number of plateletpheresis donors, donations, and units before and after the ban

\begin{tabular}{|c|c|c|c|c|}
\hline & \multicolumn{4}{|l|}{ Mean (SD) } \\
\hline & Before the ban & After the ban & $t$ & pa \\
\hline \multicolumn{5}{|c|}{ Overall discovery set } \\
\hline Total \#donors & 10297 (1442) & 7530 (934) & 3.10 & 0.0092 \\
\hline Total \#donations & 17744 (2108) & 21662 (3753) & 2.45 & 0.0308 \\
\hline Total units $(U)$ & 28781 (3959) & 38425 (7417) & 3.14 & 0.0085 \\
\hline \multicolumn{5}{|c|}{ Overall replicate set } \\
\hline Total \#donors & $6170(975)$ & 4754 (317) & 2.42 & 0.0325 \\
\hline Total \#donations & 9349 (1843) & 12469 (392) & 2.83 & 0.0150 \\
\hline Total units (U) & $12803(2250)$ & 20558 (1488) & 5.56 & 0.0001 \\
\hline
\end{tabular}

Table 3 demonstrated that both average apheresis platelet units per donor and average total number of plateletpheresis donations per donor after the ban were significantly higher than those before the ban for all covariates including gender, age, and blood donation history in both datasets(all p-values $<0.05$ ). Further comparisons revealed that male donors or donors with plateletpheresis donation history had significantly larger increase (i.e., after-before mean difference) of both average apheresis platelet units per donor and average total number of plateletpheresis donations per donor compared to their peer groups (i.e., female donors or donors with other donation history) (all p-values < 0.05). 
Table 3

Comparisons of average plateletpheresis donations/units per donor before and after the ban for individuals

Average plateletpheresis Donations Per Donor

Before After Ban

Ban

Mean

(SD), $\mathbf{n}$
Mean

(SD), $\mathrm{n}$

\section{Mean Difference $\quad t$}

Average apheresis platelet Units Per Donor

Before After Ban

Ban

Mean

(SD), $n$

\section{Mean}

(SD), $\mathrm{n}$
(SE)

\section{Overall discovery}

set

\begin{tabular}{|c|c|c|c|c|c|c|c|c|c|c|}
\hline Total & $1.7(0.1), 11$ & $2.9(0.2), 3$ & 1.1(0.1) & 9.19 & 0.0096 & $2.8(0.1), 11$ & $5.1(0.4), 3$ & $2.3(0.1)$ & 9.60 & 0.0097 \\
\hline \multicolumn{11}{|l|}{ Gender } \\
\hline male & $1.9(0.1), 11$ & $3.2(0.2), 3$ & $1.3(0.1)$ & 13.17 & 0.0041 & $3.1(0.1), 11$ & $5.7(0.4), 3$ & $2.6(0.2)$ & 10.90 & 0.0074 \\
\hline female & $1.4(0.1), 11$ & $1.9(0.1), 3$ & $0.6(0.1)$ & 11.28 & <. 0001 & $2.0(0.1), 11$ & $3.1(0.3), 3$ & $1.1(0.1)$ & 7.09 & 0.0164 \\
\hline \multicolumn{11}{|l|}{ Age } \\
\hline$\leq 35$ years & $1.6(0.1), 11$ & $2.6(0.2), 3$ & $1.0(0.1)$ & 11.62 & 0.0057 & $2.6(0.1), 11$ & $4.6(0.4), 3$ & $2.1(0.2)$ & 8.53 & 0.0120 \\
\hline > 35 years & $2.2(0.1), 11$ & $3.5(0.2), 3$ & $1.3(0.1)$ & 10.99 & 0.0047 & $3.7(0.3), 11$ & $6.4(0.5), 3$ & $2.7(0.3)$ & 9.89 & 0.0054 \\
\hline \multicolumn{11}{|c|}{$\begin{array}{l}\text { Blood donation } \\
\text { historya }\end{array}$} \\
\hline WB & $\begin{array}{l}1.2(< \\
0.1), 11\end{array}$ & $\begin{array}{l}1.9(< \\
0.1), 3\end{array}$ & $0.7(<0.1)$ & 55.9 & $\begin{array}{l}<.0001 \\
0.00\end{array}$ & $1.8(0.1), 11$ & 3.1(0.1),3 & $1.3(0.1)$ & 18.46 & 0.0009 \\
\hline PLT & $2.6(0.1), 11$ & $3.6(0.2), 3$ & $1.0(0.1)$ & 10.25 & 0.0080 & $4.4(0.2), 11$ & $6.6(0.4), 3$ & $2.2(0.2)$ & 10.32 & 0.0063 \\
\hline Both & 3.3(0.2),11 & $4.0(0.2), 3$ & $0.7(0.1)$ & 6.27 & 0.0049 & $5.9(0.3), 11$ & $7.3(0.5), 3$ & $1.5(0.3)$ & 5.18 & 0.0206 \\
\hline None & $1.2(<$ & $1.6(0.2), 3$ & $0.5(0.1)$ & 5.16 & 0.0320 & $1.7(0.1), 11$ & $2.6(0.3), 3$ & $0.9(0.2)$ & 5.95 & 0.0245 \\
\hline
\end{tabular}

FRD:

$0.1), 11$

FRD

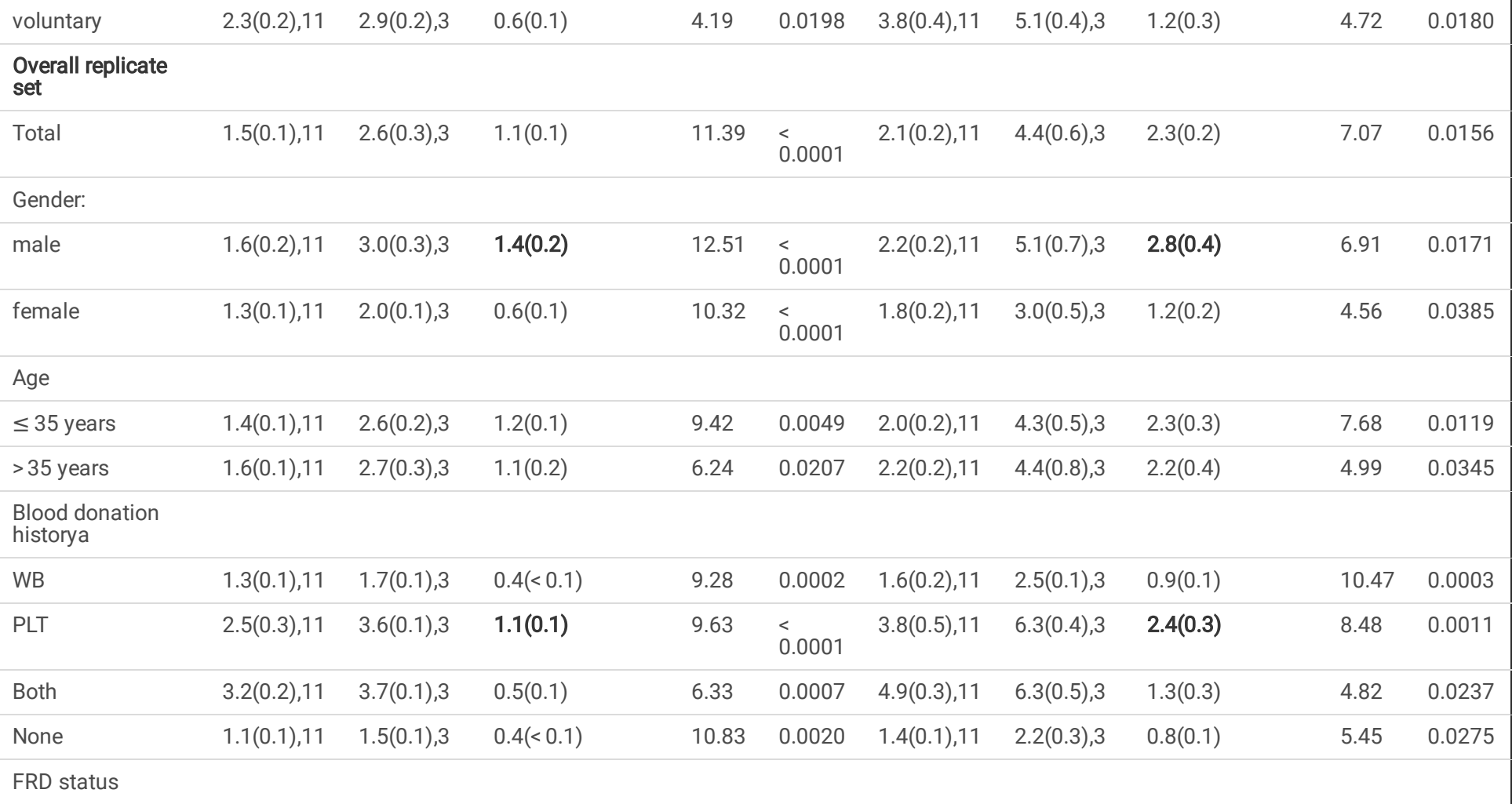

Bold font indicates statistical significance for the indicated group vs. other group(s) within the same variable at the $a=0.05$ level. Z-test was used to compare two mean differences: $Z=$ (mean difference1-mean difference2)/sqrt(se12 + se22), two-tailed.

a"None"=no blood donation history; "WB"=whole blood donation history only; "PLT"=plateletpheresis donation history only; "Both"=both whole blood and plateletpheresis donations history. 


$\begin{array}{lllllllllll}\text { FRD } & \text { NA } & \text { NA } & \text { NA } & \text { NA } & \text { NA } & \text { NA } & \text { NA } & \text { NA } & \text { NA } & \text { NA } \\ \text { voluntary } & 1.9(0.3), 11 & 2.6(0.3), 3 & 0.7(0.2) & 4.00 & 0.0136 & 2.8(0.5), 11 & 4.4(0.6), 3 & 1.6(0.4) & 4.43 & 0.0221\end{array}$

Bold font indicates statistical significance for the indicated group vs. other group(s) within the same variable at the $a=0.05$ level. Z-test was used to compare two mean differences: $Z=$ (mean difference1-mean difference2)/sqrt(se12 + se22), two-tailed.

a"None"=no blood donation history; "WB"=whole blood donation history only; "PLT"=plateletpheresis donation history only; "Both"=both whole blood and plateletpheresis donations history.

In addition, in terms of average apheresis platelet units per donor among family/replacement donors, voluntary donors before the ban, and voluntary donors after the ban, the family/replacement donors contributed the least, voluntary donors before the ban the second, and voluntary donors after the ban the most (ANOVA with post-hoc Tukey tests, all p-values $<0.0001$ )(Additional file 5).

\section{Model-based trend of average apheresis platelet units per donor over time in overall GZ set}

Table 4 summarized the characteristics of two independent pseudo-panel datasets (overall GZ set: $n=330$ cells; overall CD set: $n=316$ cells) with the number of plateletpheresis donors per cell $\geq 30$. Figure $1 \mathrm{~A}$ and Fig. 1B showed24 individual trajectories of the average apheresis platelet units per donor representing 24 cohort groupswith each who shared common gender, birth year, and blood donation history over 14 cross-sections in the overall GZ and CD pseudo-panel sets, respectively. Figure $1 \mathrm{C}$ and Fig. 1D visualized an overall mean profile of the outcome over time in the GZ and CD sets, respectively, indicating that there was a breakpoint at the 11th cross-section (just right before the ban) with a roughly horizontal line to the left of the breakpoint and significantly positive linear trend to the right. 
Table 4

Characteristics of two independent pseudo-panel datasets(the number of plateletpheresis donors per cell $\geq 30$ )

\begin{tabular}{|c|c|c|c|c|c|c|}
\hline \multirow[t]{2}{*}{ Gender } & \multirow[t]{2}{*}{ Birth year } & \multicolumn{4}{|l|}{ Blood donation historya } & \multirow[t]{2}{*}{ Total } \\
\hline & & None & WB & PLT & Both & \\
\hline & & Number of cross-sections & Number of cross-sections & Number of cross-sections & Number of cross-sections & \\
\hline & & $($ Min, Max)b & (Min, Max) & (Min, Max) & (Min, Max) & \\
\hline \multicolumn{7}{|c|}{ Overall GZ set } \\
\hline Male & 1952-1974 & $14(63,489)$ & $14(79,140)$ & $14(156,234)$ & $14(220,338)$ & 56 \\
\hline Male & $1975-1984$ & $14(114,1051)$ & $14(158,352)$ & $14(312,435)$ & $14(281,460)$ & 56 \\
\hline Male & $1985-2001$ & $14(897,3528)$ & $14(329,947)$ & $14(737,1478)$ & $14(356,1226)$ & 56 \\
\hline Female & $1952-1974$ & $11(79,237)$ & $11(30,55)$ & $14(51,80)$ & $14(42,77)$ & 50 \\
\hline Female & 1975-1984 & $14(55,425)$ & $14(41,102)$ & $14(53,87)$ & $14(36,63)$ & 56 \\
\hline Female & 1985-2001 & $14(409,1396)$ & $14(191,408)$ & $14(246,357)$ & $14(149,443)$ & 56 \\
\hline Total & & 81 & 81 & 84 & 84 & 330 \\
\hline \multicolumn{7}{|c|}{ Overall CD set } \\
\hline Male & 1952-1974 & $14(51,648)$ & $14(44,173)$ & $14(73,115)$ & $14(91,174)$ & 56 \\
\hline Male & 1975-1984 & $14(79,807)$ & $14(47,144)$ & $14(98,151)$ & $14(89,154)$ & 56 \\
\hline Male & 1985-2001 & $14(585,2481)$ & $14(148,440)$ & $14(207,851)$ & $14(132,475)$ & 56 \\
\hline Female & $1952-1974$ & $14(79,318)$ & $11(31,160)$ & $12(32,71)$ & $12(30,125)$ & 49 \\
\hline Female & 1975-1984 & $14(57,279)$ & $14(36,70)$ & $10(32,55)$ & $5(30,67)$ & 43 \\
\hline Female & 1985-2001 & $14(398,975)$ & $14(75,230)$ & $14(76,286)$ & $14(48,201)$ & 56 \\
\hline Total & & 84 & 81 & 78 & 73 & 316 \\
\hline
\end{tabular}

Thus, to the overall GZ set, we fitted a two-piecewise linear mixed-effects model in which we specified intercept as the random term with an unstructured covariance-structure. The covariate birth year was excluded due to its non-significance. As shown in Table 5 and Fig. $2 \mathrm{~A}$, the final model presented a twopiecewise linear trend of average apheresis platelet units per donor over time (i.e., cross-section) with a horizontal line to the left of the breakpoint ( $\beta_{\text {timeBefore11 }}$ $=0.0111, p=0.0976)$ and a significantly positive linear trend to the right $\left(\beta_{\text {timeAfter11 }}=0.0404, p<0.0001\right)$. This result suggests that the average apheresis platelet units per donor were maintained at lower level and did not change with time before the ban, but started increasing linearly with time after the ban. 
Table 5

Final models derived from two independent overall pseudo-panel datasets

\begin{tabular}{|c|c|c|c|c|c|c|}
\hline & \multicolumn{3}{|c|}{ Overall GZ set ${ }^{a}$} & \multicolumn{3}{|c|}{ Overall CD set ${ }^{b}$} \\
\hline & $\beta$ & SE & $\mathrm{p}$ & $\beta$ & SE & $\mathbf{p}$ \\
\hline Intercept & 0.8636 & 0.4643 & 0.0782 & 0.9637 & 0.2180 & 0.0002 \\
\hline timeBefore11 & 0.0111 & 0.0067 & 0.0976 & 0.0077 & 0.0084 & 0.3582 \\
\hline timeAfter 11 & 0.0404 & 0.0093 & $<0.0001$ & 0.0441 & 0.0102 & $<0.0001$ \\
\hline \multicolumn{7}{|l|}{ Gender } \\
\hline male vs. female & 1.3370 & 0.4138 & 0.0044 & 0.7449 & 0.1909 & 0.0009 \\
\hline \multicolumn{7}{|l|}{ Blood donation historyc } \\
\hline WB vs. None & 0.1790 & 0.5852 & 0.7630 & 0.1928 & 0.2687 & 0.4816 \\
\hline PLT vs. None & 2.6243 & 0.5851 & 0.0003 & 2.1231 & 0.2690 & $<0.0001$ \\
\hline Both vs. None & 3.9440 & 0.5851 & $<0.0001$ & 3.2293 & 0.2705 & $<0.0001$ \\
\hline \multicolumn{7}{|l|}{ timeAfter11 $\times$ gender } \\
\hline time $\times$ male vs. time $\times$ female & 0.0550 & 0.0072 & $<0.0001$ & 0.0325 & 0.0086 & 0.0002 \\
\hline \multicolumn{7}{|l|}{ timeAfter $11 \times$ history } \\
\hline time $\times$ WB vs. time $\times$ none & 0.0331 & 0.0105 & 0.0018 & 0.0069 & 0.0120 & 0.5655 \\
\hline time $\times$ PLT vs. time $\times$ none ${ }^{d}$ & 0.0698 & 0.0104 & $<0.0001$ & 0.1373 & 0.0120 & $<0.0001$ \\
\hline time $\times$ Both vs. time $\times$ none ${ }^{d}$ & 0.0444 & 0.0104 & $<0.0001$ & 0.0507 & 0.0120 & $<0.0001$ \\
\hline \multicolumn{7}{|c|}{ 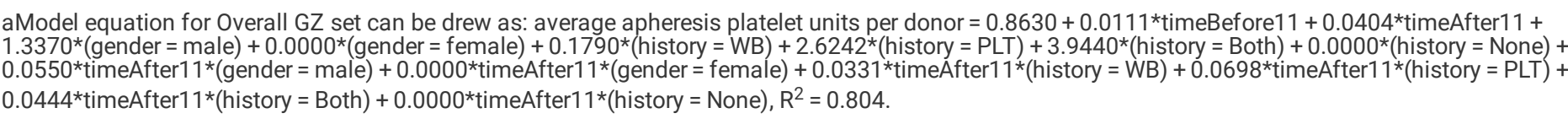 } \\
\hline \multicolumn{7}{|c|}{ 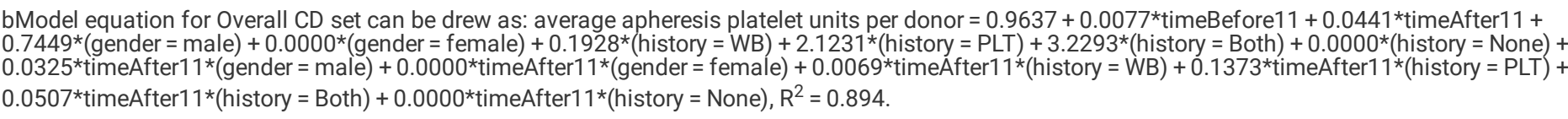 } \\
\hline \multicolumn{7}{|c|}{$\begin{array}{l}\text { c"None"=no blood donation history; "WB"=whole blood donation history only; "PLT"=plateletpheresis donation history only; "Both"=both whole blood and } \\
\text { plateletpheresis donations history. }\end{array}$} \\
\hline \multicolumn{7}{|c|}{$\begin{array}{l}\text { dThe difference of the outcome change between time }{ }^{\star} P L T \text { and time } e^{\star} \text { Both was tested using } Z=a b s(\beta 2-\beta 1) / S Q R T(S E 12+S E 22) \text {, } a=0.05 \text {, one-tailed. For } \\
\text { overall GZ set: } Z=1.727, p=0.0421 \text {; for overall } C D \text { set: } Z=5.103, p<0.0001 \text {. }\end{array}$} \\
\hline
\end{tabular}

In the model, independent covariates measured whether baseline outcome differed by group. Table 5 demonstrated thaton average at baseline, male donated 1.337 more apheresis platelet units per donor than female $\left(\beta_{\text {male vs female }}=1.3370, p=0.0044\right)$, and the donated apheresis platelet units per donor was significantly higher in the donors who had platelet donation history than that in their peers who had no blood donation history $\left(\beta_{\mathrm{PLT}} \mathrm{vs}\right.$ None $=2.6243, p=0.0003$; $\left.\beta_{\text {Both vs None }}=3.9440, p<0.0001\right)$ whereas donations by the donors with whole blood donation history only were not significant $\left(\beta_{\text {WB vs None }}=0.1790, p=\right.$ 0.7630). Due to the non-significance of the slope for the timeBefore 11 term, we only considered the interactions between the timeAfter 11 and covariates in the model. After the model selection, a two-way interaction term gender*blood donation history and a three-way interaction term timeAfter $11^{*}$ gender*blood donation history were excluded due to their non-significance. The regression coefficient of the interaction term measured whether the outcome change differed by covariate-specific groups. As shown in Table 5, on average after the ban, the outcome change was significant in males than that in females ( $\beta_{\text {timexmale }}$ timexfemale $=0.0550, p<0.0001)$ and in donors with blood donation history than that in their peers without donation history $\left(\beta_{\text {timexwB vs timexnone }}=0.0331, p=\right.$ $0.0018 ; \beta_{\text {timexPLT vs timexnone }}=0.0698, p<0.0001$; and $\beta_{\text {timexBoth vs timexnone }}=0.0444, p<0.0001$ ). These results suggest that the contributions of gender and blood donation history to the model had heterogeneity for both the baseline outcome value and the outcome change after the ban.

The Pearson residual plots, either marginally for the consideration of fixed effects only or conditionally for the consideration of both fixed and random effects, for the final model indicated that the model's assumptions - normality and heteroscedasticity were not significantly violated (Additional file 10). Five-fold cross-validation demonstrated that the over-fitting percentage of the final model for overall GZ set accounted for only $0.28 \%$ ( Additional file 6 ), suggesting that there was no significant over-fitting issue for the final model.

Replication of the model-based trend of average apheresis platelet units per donor over time in overall CD set 
Next, we used an independent overall CD set to test the replication of the final model obtained from the overall GZ set. As shown in Table 5 and Fig. 2B, all parameters' estimates in the final model for the overall CD set were similar to those for the overall GZ set, except for that the outcome change for the donors with whole blood donation history only was not significant and that the outcome values across all time-points appeared to be systematically reduced in the overall CD set, compared to those in the overall GZ set. The latter result was consistent with that from the individual-level data, i.e., the overall average apheresis platelet units per donor in Guangzhou (overall mean $=3.3 \mathrm{U}, \mathrm{SD}=1.0$ ) was higher than that in Chengdu (overall mean $=2.6 \mathrm{U}, \mathrm{SD}=1.0$ ) with a marginally non-significance (mean difference $=0.7 \mathrm{U}, 95 \% \mathrm{Cl}-0.04-1.5, \mathrm{p}=0.0644$, Additional file 5 ). The assumptions of normality and heteroscedasticity were not significantly violated (Additional file 10), and the over-fitting percentage of the final model for overall CD set was $2.52 \%$ (Additional file 6 ). These results suggest that the two-piecewise linear trend of the average apheresis platelet units per donor over time with the cross-section in which the ban went into effect as a time breakpoint that we observed using overall GZ set can be replicated using an independent dataset - overall CD set with some minor variations.

\section{Effect of family/replacement platelet donations on model-based trend of the average apheresis platelet units per donor over 14 cross-sections in both $\mathrm{GZ}$ and CD sets}

After removing the FRD across the first 11 cross-sections followed by re-grouping the data (Additional file 7),our modeling results demonstrated that although the average apheresis platelet units per donor still significantly increased over time after the ban ( $\beta$ timeAfter $11=0.0588$ and 0.0583 for voluntary $G Z$ and $C D$ subsets, respectively, all p-values $<0.0001)$, a positive linear trend of the outcome values over time before the ban also became significant $(\beta$ timeAfter $11=$ 0.0400 and 0.0499 for voluntary GZ and CD subsets, respectively, all p-values < 0.0001 ) (Additional file 8 and Fig. 3). These results suggest that the FRD is a critical factor to influence the trend of the outcome values over time before the ban. The heterogeneity for group-specific baseline outcome and outcome change after the ban was still similar to that from the overall GZ and CD sets with some variations (Additional file 8). Neither significant violations of the model's assumptions (Additional file 11) nor over-fitting issue (over-fitting percentage was $4.64 \%$ for voluntary GZ subset and $0.65 \%$ for voluntary CD subset) (Additional file 9) were found for the final models.

\section{Discussion}

In the present study, we observed that total number of donations and total apheresis platelet units after the ban were significantly higher than those before the ban whereas total number of plateletpheresis donors showed the opposite in both discovery and replicate datasets. These findings quantitatively confirmed an emerging phenomenon of "fewer donors, more blood" that we preliminarily observed in the field since the implement of the FRD ban in China, which breaks the belief of "more donors, more blood" in the past plateletpheresis donation practice.

Furthermore, using a pseudo-panel data approach, we observed that the average apheresis platelet units per donor presented a horizontal line over time before the ban, and then followed a significantly positive linear trend over time after the ban from both discovery and replicate datasets. To our knowledge, this study is the first to report such a two-piecewise linear trend of the average apheresis platelet units per donor over time before and after a nationwide FRD ban.

Our modeling results also revealed that male donors and donors with plateletpheresis donation history had an increased baseline outcome and a significant outcome change over time after the ban. These findings are consistent with those from our individual-level data, i.e., both male donors and donors with plateletpheresis donation history had significantly larger increases of both average apheresis platelet units per donor and average total number of plateletpheresis donations per donor compared to their peer groups (i.e., female donors or donors with other donation history), which may be defined as improved plateletpheresis donation behavior. Therefore, a possible mechanism underlying the two-piecewise linear trend of the outcome over time before and after the ban could be the FRD ban-related improved plateletpheresis donation behavior. Evidence showed that the improved donation behavior can be related to the increased altruism[21,22] and social responsibility of blood donors[21] whereas repeat donors had a higher return donation rate with altruistic reasons[22] .On the other hand, studies also demonstrated that male donors more frequently donated blood[22, 23] and women were less likely to donate blood[24, 25] probably due to the pregnancy- and/or lactation-based absence [24]. However, whether the ban can increase altruism and social responsibility of male donors and/or repeat donors is unknown. Another possible mechanism could be directly related to the implement of the FRD ban. Our findings indicated that compared to voluntary plateletpheresis donors, family/replacement donors donated significantly smaller volume of apheresis platelet, and therefore, removing family/replacement donors from the data led to the trend of outcome over time before the ban being changed from a horizontal line to a weak and positive linear line. Taken together, the two-piecewise linear trend of outcome over time may be an integrative result from two ban-related factors: the implement of the FRD ban and the improved donation behavior of male donors and/or donors with platelet donation history after the ban.

Systematic reduction of the outcome for the model as a whole in the samples from Chengdu Blood Center compared to that from Guangzhou Blood Center is consistent with our previous study, in which we reported that the average blood donation volume per resident was higher in Guangdong, whose capital city is Guangzhou, than that in Sichuan, whose capital city is Chengdu (3.06U for Guangdong vs. $2.56 \mathrm{U}$ for Sichuan) [26] .

Our study has several strengths. The use of an independent external dataset to replicate the findings from the discovery dataset significantly increased the external validity of our findings. The internal validity of our results was improved by the application of five-fold cross-validation. As mentioned above, our datasets presented a pseudo-panel structure, thus, the use of a pseudo-panel data approach maximized the reliability and validity of our analysis. Our study also has some limitations. Only three covariates were available for our modeling in both discovery and replicate datasets, thus, we cannot completely rule out the possible confounding effects of the unmeasured covariates. However, by calculating the linear mixed- effects model's R-square values(1-SSE/(SSE + SSR)) [27], about $80-89 \%$ of the variance for the outcome variable can be explained by the independent variables that are included in our final models in overall GZ and CD datasets, respectively (Table 5). The nationwide FRD ban in China was effective on April 1, 2018, thus, the number of the cross-sections available after the ban was relatively few and further continuously monitoring the trend of the outcome over time after the ban is needed. Our study was a cross-sectional design, thus, couldn't figure out the causal relationship between the covariates and the outcome. Pseudo-panel data approach is an aggregation method, therefore, we lost some information and statistical power. 


\section{Conclusion}

In conclusion, the significant increase of the average apheresis platelet units per donor over time after the FRD ban may be related to the implement of the FRD ban and the improved donation behavior of male donors and/or donors with platelet donation history after the ban. Our findings suggest that to further increase the plateletpheresis donations in China, a continuous implement of the FRD ban is encouraged and more rigorous blood donation motivations are needed for those donors who are female and who have no donation history. They may also potentially motivate the policymakers in other countries where the FRD for plateletpheresis donation is still legitimate to phase out their FRD strategy and ultimately achieve $100 \%$ voluntary plateletpheresis donation.

\section{Abbreviations}

FRD: family/replacement donation

STROBE: Strengthening the Reporting of Observational Studies in Epidemiology

GZ: Guangzhou

CD: Chengdu

GZBC: Guangzhou Blood Center

CDBC: Chengdu Blood Center

\section{Declarations}

\section{Ethics declarations}

\section{Ethics approval and consent to participate}

Not applicable.

\section{Consent for publication}

Not applicable.

\section{Availability of data and materials}

The datasets used during this study will be made available by the investigators on reasonable request.

\section{Competing interests}

The authors declare that they have no competing interests.

\section{Funding}

This work was supported by the Guangzhou Municipal Science and Technology Project (No. 202002030447) and the Weigao Fund of the Chinese Society of Blood Transfusion (No. CSBT-WG-2019-06).

\section{Authors' Contribution}

JC,YF, and GZ conceived and designed the study. S L,HC,YL, and JK were responsible for the data collection used in this study from Guangzhou Blood Center or Chengdu Blood Center. LZ and YL exported the used data from the information system in Guangzhou Blood Center and Chengdu Blood Center, respectively. YF and XF supervised and coordinated all aspects of the study in Guangzhou Blood Center and Chengdu Blood Center, respectively. GZ supervised all aspects of data analysis. JC and GZ implemented the whole process of data analysis and wrote the first draft. All authors contributed to data interpretation, critical revision of the manuscript, and approved the final version of the manuscript. JC is guarantor. The corresponding authors attest that all listed authors meet authorship criteria and that no others meeting the criteria have been omitted.

\section{Acknowledgements}

We would like to thank Guangdong Pass Soft Medical Technology Co., Ltd and Tangshan Qiao Technology Co., Ltd, the providers of the blood service information system for Guangzhou Blood Center and Chengdu Blood Center, respectively, for their assistance in data extraction. We also want to thank Professor Lin Xu at the Sun Yat-sen University School of Public Health for her valuable advice at the early stage of the study design.

\section{References}

1. Watkins NA, Dobra S, Bennett P, Cairns J, Turner ML. The management of blood safety in the presence of uncertain risk: a United kingdom perspective. Transfus Med Rev. 2012;26(3):238-251. 
2. Custer B, Johnson ES, Sullivan SD, et al. Community blood supply model: development of a new model to assess the safety, sufficiency, and cost of the blood supply. Medical decision making : an international journal of the Society for Medical Decision Making. 2005;25(5):571-582.

3. Allain JP, Sibinga CT. Family donors are critical and legitimate in developing countries. Asian J Transfus Sci. 2016;10(1):5-11.

4. Kimani D, Mwangi J, Mwangi M, et al. Blood donors in Kenya: a comparison of voluntary and family replacement donors based on a population-based survey. Vox Sang. 2011;100(2):212-218.

5. WHO. Towards 100\% voluntary blood donation-A global framework for action. 2010; https://www.who.int/bloodsafety/publications/9789241599696/en/. Accessed March 20, 2020.

6. Jenny H, Saluja S, Sood R, et al. Access to safe blood in low-income and middle-income countries: lessons from India. BMJ Global Health. 2017;2(2).

7. Asenso-Mensah K, Achina G, Appiah R, Owusu-Ofori S, Allain JP. Can family or replacement blood donors become regular volunteer donors? Transfusion. 2014;54(3 Pt 2):797-804.

8. Allain JP. Current approaches to increase blood donations in resource-limited countries. Transfusion medicine (Oxford, England). 2019;29(5):297-310.

9. WHO. Blood safety and availability. 2019; https://www.who.int/en/news-room/fact-sheets/detail/blood-safety-and-availability. Accessed 14 Octber, 2019.

10. Ping H, Xing N. Blood shortages and donation in China. The Lancet. 2016;387(10031):1905-1906.

11. Xue R, Chen Y, Wen J. Blood shortages and donation in China. The Lancet. 2016;387(10031):1905.

12. Ugwu AO, Madu AJ, Efobi CC, Ibegbulam OG. Pattern of blood donation and characteristics of blood donors in Enugu, Southeast Nigeria. Nigerian journal of clinical practice. 2018;21(11):1438-1443.

13. Lebo MJ, Weber C. An Effective Approach to the Repeated Cross-Sectional Design. American Journal of Political Science. 2015;59(1):242-258.

14. Obisesan O, Thompson E, Obisesan A, Akinola O, Commodoremensah Y. Association between Non-Cigarette/Smokeless Tobacco and Hypertension in the National Health Interview Survey: A Pseudo-Panel Analysis. International Journal of Public Health Science. 2016;5(1):60-69.

15. Saksena M, Maldonado N. A Dynamic Estimation of Obesity Using Nhanes Data: A Pseudo-Panel Approach. Health Economics. 2017;26(12):140-159.

16. Tsai CP, Mulley C, Clifton G. A Review of Pseudo Panel Data Approach in Estimating Short-run and Long-run Public Transport Demand Elasticities. Transport Reviews. 2014;34(1):102-121.

17. Meng Y, Brennan A, Purshouse R, et al. Estimation of own and cross price elasticities of alcohol demand in the UK-A pseudo-panel approach using the Living Costs and Food Survey 2001-2009. Journal of health economics. 2014;34(100):96-103.

18. von Elm E, Altman DG, Egger M, Pocock SJ, Gøtzsche PC, Vandenbroucke JP. [The Strengthening the Reporting of Observational Studies in Epidemiology [STROBE] statement: guidelines for reporting observational studies]. Gaceta sanitaria. 2008;22(2):144-150.

19. Anowar S, Eluru N, Mirandamoreno LF. Analysis of Vehicle Ownership Evolution in Montreal, Canada Using Pseudo Panel Analysis. Transportation. 2016;43(3):531-548.

20. Wu S. Two-piecewise Random Coefficient Model Using Proc Mixed. 2010; https://www.lexjansen.com/pharmasug/2010/SP/SP03.pdf. Accessed April 20, 2020.

21. Sojka BN, Sojka P. The blood donation experience: self-reported motives and obstacles for donating blood. Vox Sanguinis. 2007;94(1):56-63.

22. Kasraian L, Hosseini S, Dehbidi S, Ashkaniesfahani S. Return rate in blood donors: A 7-year follow up. Transfusion Medicine. 2019.

23. Kasraian L, Tavassoli A. Relationship between first-year blood donation, return rate for subsequent donation and demographic characteristics. Vox Sanguinis. 2012;10(4):448-452.

24. Misje AH, Bosnes V, Heier HE. Gender differences in presentation rates, deferrals and return behaviour among Norwegian blood donors. Vox Sanguinis. 2010;98.

25. Wittock N, Hustinx L, Bracke P, Buffel V. Who donates? Cross-country and periodical variation in blood donor demographics in Europe between 1994 and 2014. Transfusion. 2017;57(11):2619-2628.

26. Chen J, Ouyang J, Xie G, et al. Problems and challenges: development of blood transfusion services in Mainland China within the context of health-care system reform. Transfusion Medicine. 2019;29(4):253-261.

27. Nakagawa S, Schielzeth $\mathrm{H}$. A general and simple method for obtaining R2 from generalized linear mixed-effects models. Methods in Ecology and Evolution. 2013;4(2):133-142.

\section{Figures}


Figure 1.
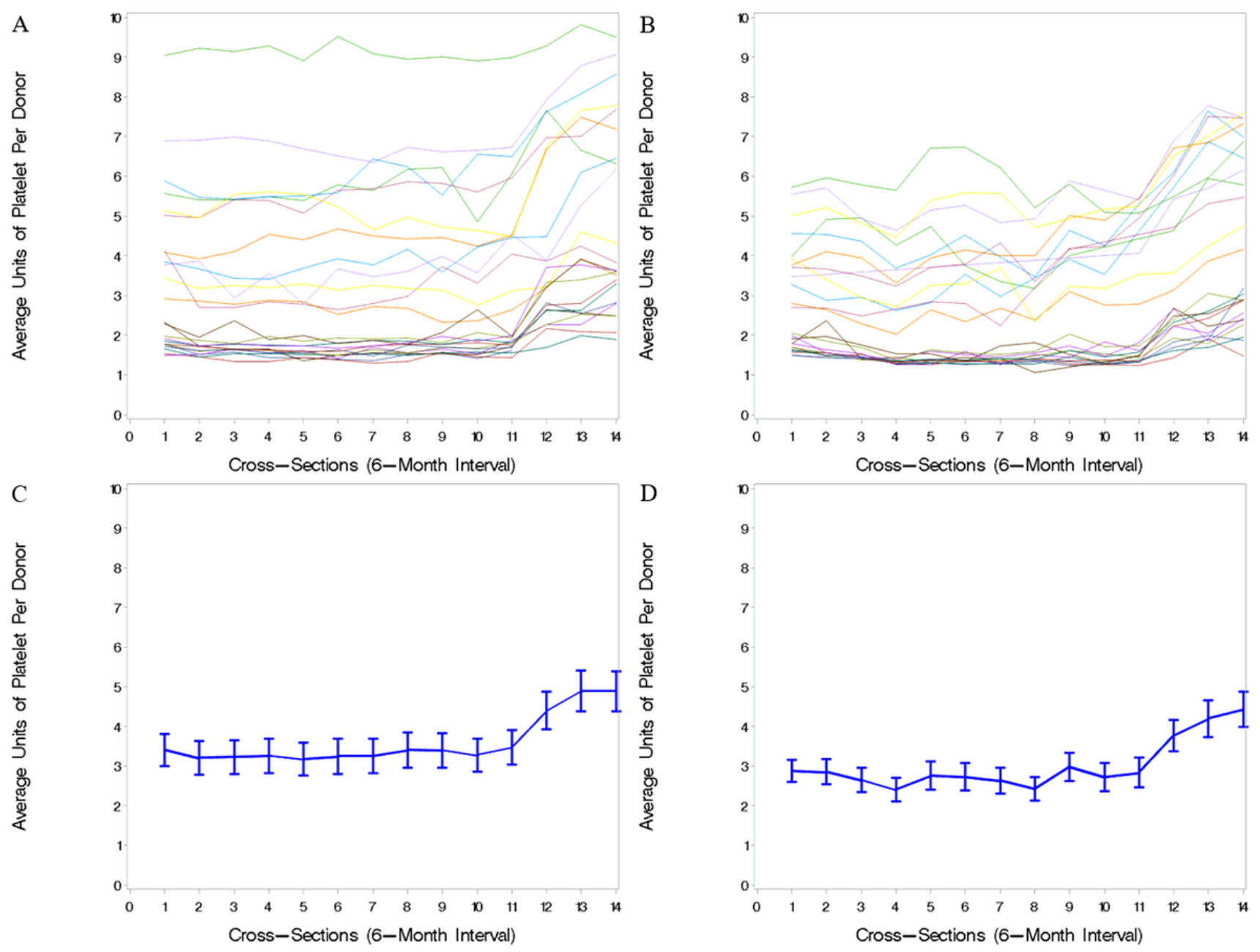

\section{Figure 1}

The trend of average apheresis platelets units per donor over time for overall pseudo-panel datasets. Panels A and B visualize individual cohort group trajectories (outcome vs. time) with each line representing a trajectory of a cohort group who had common gender, birth year, and blood donation history for overall GZ and CD pseudo-panel datasets, respectively; panels C and D present the trend of overall mean outcome over time for overall GZ and CD pseudopanel datasets, respectively.

Figure 2.
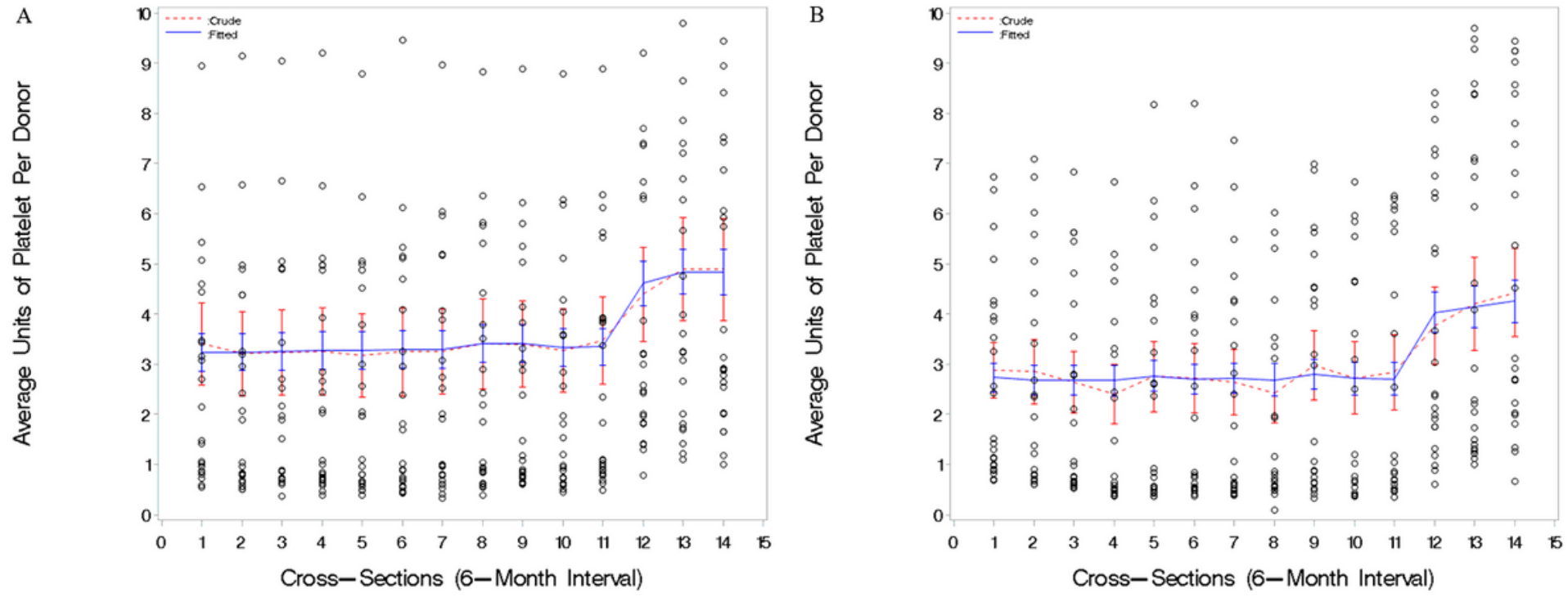

Figure 2 
Visualization of the final two-piecewise linear mixed effects models from overall pseudo-panel datasets. Panels A and B visualize how close both the fitted and the crude trends of outcome mean over time are for overall GZ and CD pseudo-panel datasets, respectively. The dotted line represents the crude trend and the solid line the fitted trend. The vertical lines depict standard errors of the outcome means. The scattered circles are raw pseudo-panel data.

Figure 3.
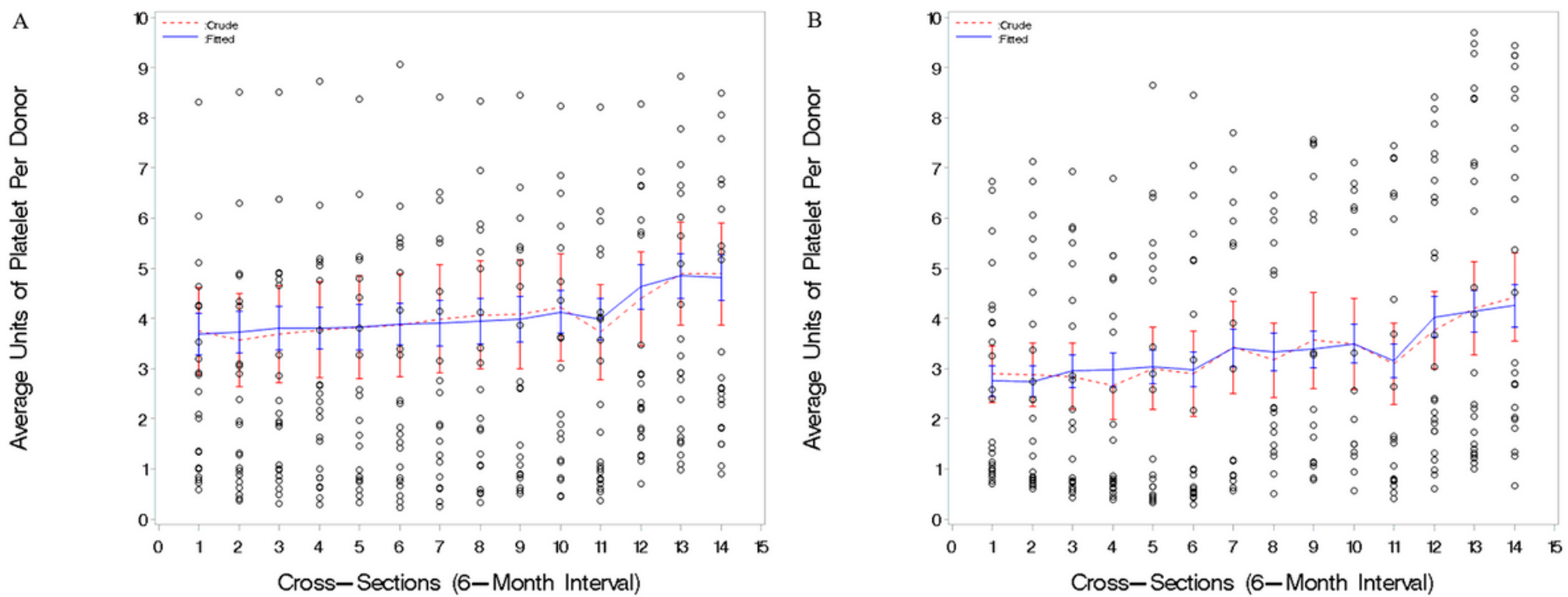

\section{Figure 3}

Visualization of the final two-piecewise linear mixed effects models from voluntary pseudo-panel subsets. Panels A and B visualize how close both the fitted and the crude trends of outcome mean over time are for voluntary GZ and CD pseudo-panel subsets, respectively. The dotted line represents the crude trend and the solid line the fitted trend. The vertical lines depict standard errors of the outcome means. The scattered circles are raw pseudo-panel data.

\section{Supplementary Files}

This is a list of supplementary files associated with this preprint. Click to download.

- Additionalfile11.docx

- Additionalfile10.docx

- Additionalfile9.docx

- Additionalfile8.docx

- Additionalfile7.docx

- Additionalfile6.docx

- Additionalfile5.docx

- Additionalfile4.docx

- Additionalfile3.docx

- Additionalfile2.docx

- Additionalfile1STROBEStatement.doc 